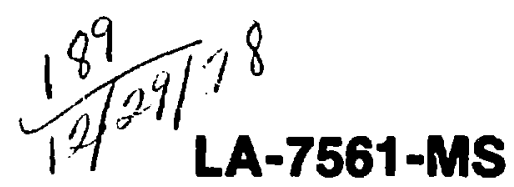

Informal Report

DR.2072 MASIER

\title{
Preprocessing of PHERMEX Flash \\ Radiographic Images with Haar and Adaptive Filtering
}

U.

票

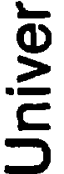


LA-7561-MS

Informal Report

UC-32

Issued: November 1978

\title{
Preprocessing of PHERMEX Flash Radiographic Images with Haar and Adaptive Filtering
}

\author{
John E. Brolley
}

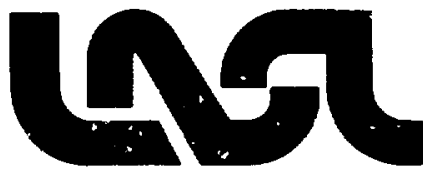


PREPROCESSING OF PHERMEX FLASH RADIOGRAPHIC IMAGES

WITH HAAR AND ADAPTIVE FILTERING

by

John E. Brolley

ABSTRACT

Work on image preparation has continued with the application of high-sequency boosting via Haar filtering. This is useful in developing line or edge structures. Widrow LMS adaptive filtering has also been shown to be useful in developing edge structure in special problems. Shadow effects can be obtained with the latter which may be useful for some problems. Combined Haar and adaptive filtering is illustrated for a PHERMEX image.

\section{INTRODUCTION}

In the first report of this series, ${ }^{1}$ several different types of operations on images were discussed and illustrative examples were presented. The present note considers additional modus operandi. High-sequency boosting via Haar filtering, histogram equalization, and adaptive filtering are applied to a PHERMEX flash radiographic image to render certain edge patterns more easily discernible.

II. HIGH-SEQUENCY BCOSTING

Haar functions constitute a complete orthonormal set with some properties rather different from the more commonly used ortionormal sets. Tutorials ${ }^{2}$ are available for details. Oniy a few introductory points will be considered here. The first eight Haar functions are displayed in Fig. 1 . In common with the Walsh functions they are defined only over a finite interval of the argument. They also show the concept of sequency. ${ }^{1,2}$ Comparison of Fig. 1 with Fig. 11 of Ref. 1, however, illuminates a striking difference. All of the Walsh functions are globally sensitive. Only the first two Haar functions are. The remaining 
Haar functions are locally sensitive. This suggests that Haar functions may be useful in edge detection and delineation. A mathematical relation for this assumption is given in Ref. 2. Fast algorithms exist ${ }^{3,4}$ which permit Haar decomposition to be done much more rapidly than Fourier.

A two-dimensional forward Haar transform of a digitized image is readily effected and transfers the image to the sequency domain. In this domain it is then possible to shift the distribution of spectral power by high-sequency boosting. ${ }^{1}$ This operation would be expected to enhance lines and edges.

As an example of the usefulness of Haar high-sequency boosting, the 512 by 512 pixei mall image used in Ref. 1 is again utilized. The original aigitized image is shown in Fig. 2 as photographed from a DIADS ${ }^{1}$ display. In Fig. 3 the effect of high-seque $\because$ boosting and low-sequency roll-off is shown using the Walsh decomposition.' The corresponding operation with Haar functions is shown in Fig. 4. In both Figs. 3 and $4, \log$ stretching ${ }^{5}$ was used.

Figures 2, 3, and 4 are not designed to permit quantitative inferences to be drawn. Nonetheless, it is clear that Haar high-sequency boosting is quite useful in emphasizing edges and liries. It is also possible to advance an untested conjecture. It will be sbserved in Fig. 3 that Walsh high-sequency boosting appears to generate artifacting that is absent or minimal in Fig. 4. A very careful scrutiny ${ }^{*}$ of Fig. 2, however, suggests that some of the checkerboarding is embedded in the original digitized picture. Since Walsh functions are globally sensitive, the ouildup of the checkerboarding in Fig. 3 may be an example of resonance. Since most of the Haar functions are not globally sensitive, the resonance (if indeed it exists) would not be expected. In Fig. 4 the checkerboarding is slightly more discernible than in Fig. 2 .

\section{ADAPTIVE FILTERING}

The raster scanning of DIADS permits the easy application of one-dimensional adaptive filtering. In this report the application of Widrow LMS adaptive filtering, ${ }^{6}$ (WLMSAF), will be considered. A future report will probably consider adaptive lattice filtering. WLMSAF is a sentient digital filter which can be instructed to learn how to drive a reasonably stationary input data sequence to

* For those wishing to do this rather assiduously, original $8-1 / 2 \times 11$ glossies of negative numbers 7730578,789196 , and 789190 may be requested from ISD-7. 
zero output. It can also be instructed to freeze its memory so that only the original signal it studied will generate zero output power.

As a first application, the DIADS image (512 by 512) of a step tablet, shown in Fig. 5, will te manipulated. The picture was first processed with Haar high-sequency boosting to produce the image displayed in Fig. 6 . The resulting image matrix was then processed line by lire. For the first 50 lines the filter was instructed to continuously learn; from 1 ine 51 on it was frozen. The result of these operations is shown in Fig. 6. Clearly, the boundary locations, which are somewhat difficult to discern in Fig. 5, have become rather more conspicuous.

As a last example, the digitized PHERMEX flash radiographic image displayed in Fig. 8 will be operated on. The first operation to be performed was histogram equalization. ${ }^{\text {** }}$ This was followed with Haar high-sequency boosting and then adaptive filtering, as in the step tablet case. The result is shown in Fig. 9. Clearly certain boundaries have become quite conspicuous.

\section{CONCLUSIONS}

Haar high-sequency boosting is clearly a useful tool for building up edge and line structure. It also requires the least computer time for this type of operation. Adaptive filtering clearly shows promise for many applications. It is possible to run the filter in many different directions, and this should be useful in some types of scene analysis. ${ }^{+}$It should be possible to implement adaptive filtering on DIADS and allow the operator to change learning rate and length in various parts of the scene as well as to freeze and thaw.

\section{ACKNOWLEDGMENTS}

R. Bagley of the Digital Image and Signal Processing group kindly performed all of the image digitizations required in this work.

\footnotetext{
¿Eastman Kodak Step Tablet No. 2, $2 i$ steps, density range 0.05 to 3.05 . ** Available in the LADIES (Los Alamos Digital Image Enhancement System) library.

${ }^{+}$ROTR90TR in the LADIES 1 ibrary can perform a variety of different rotations or reflections on the image matrix before it is passed through the adaptive filter.
} 


\section{REFERENCES}

1. J. E. Brolley, "Preprocessing of PHERMEX Flash Radiographic Images," Los Alàmos Scientific Laboratory report. LA-71G5-MS (April 1978).

2. J. E. Shore, "On the Application of Haar Functions," IEEE Trans. on Comm. 21, 209 (1973).

3. N. Anmed and R. K. Rao, Orthogonal Transforms for Digital Signal Processing (Springer Verlag, Berlin, 1975).

4. K. G. Beaucham, Walsh Functions and Their Applications (Academic Press, New York, 1977).

5. 1i. C. Andrews, "Monochrome lligita? Image Enhancement," App1. Opt. 15, 495 $(1976)$.

6. J. E. Brolley, "Preprocessing of Seismic Signals by Haar Decomposition and Adaptive Filtering for Pattern Recognition," Los Alamos Scientific Laboratory report LA-7541-MS (November 1978).

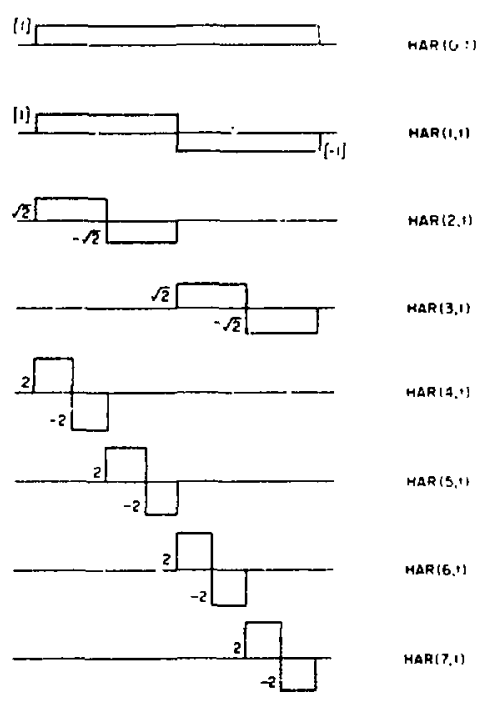

Fig. 1. The first eight Haar functions.

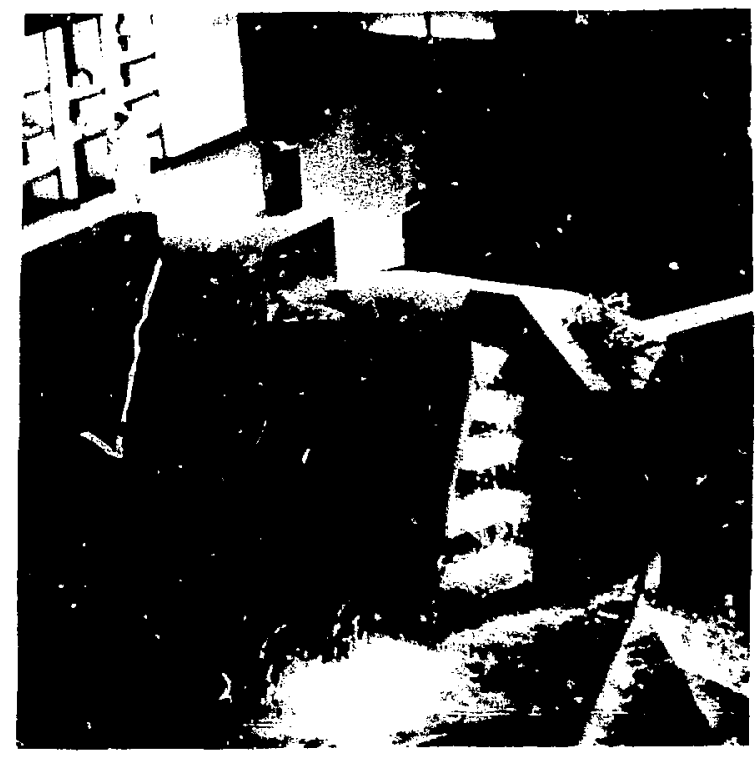

Fig. 2. Original digitized mall scene photographed from the DIADS display (512 by 517 dixeis). 


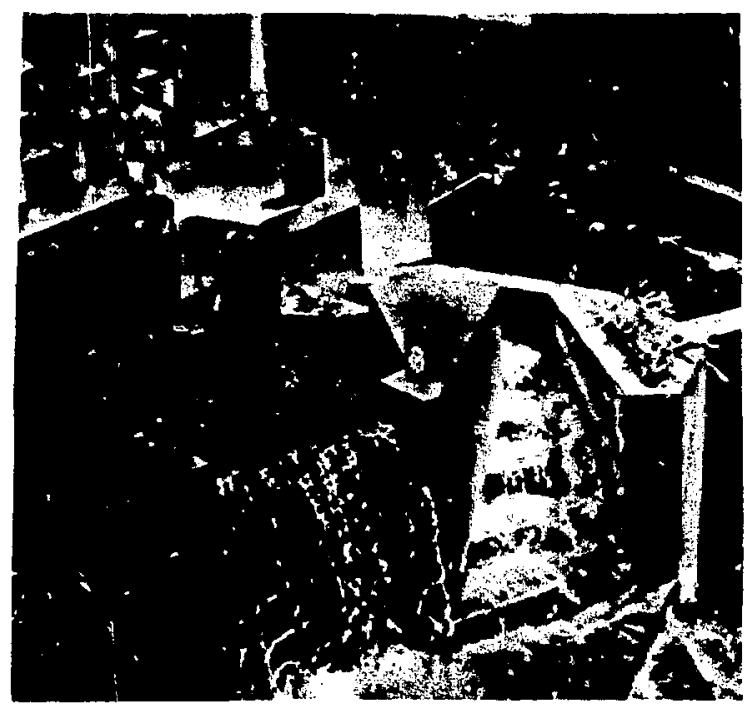

Fig. 3. Mall scene after Walsh highsequency boosting (512 by 512 pixels).

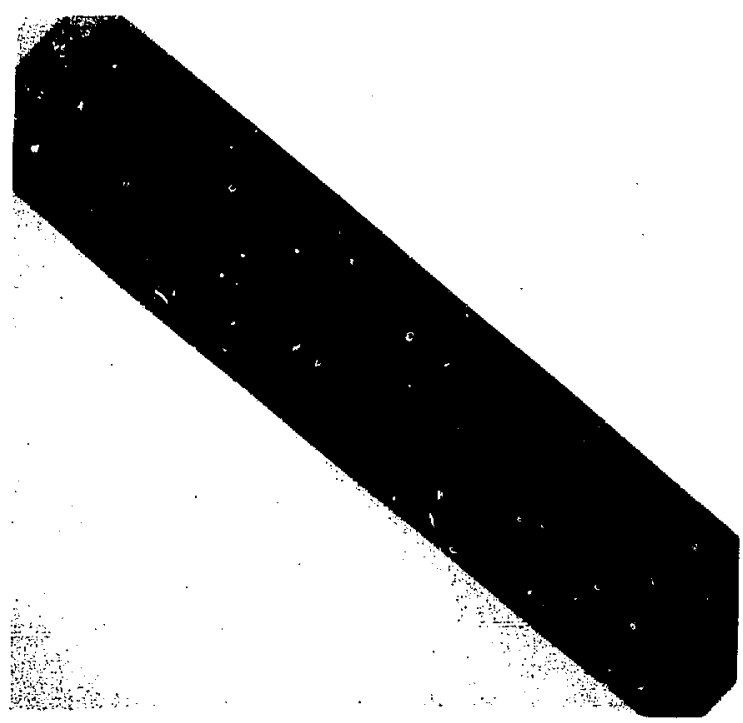

Fig. 5. Original step tabiet image ( 512 by 512 pixels).

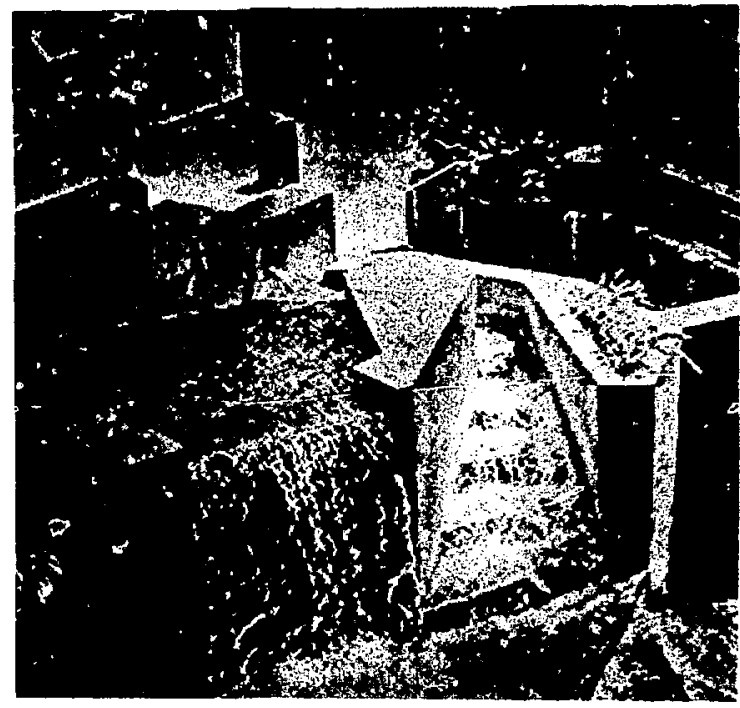

Fig. 4. Mall scene after Haar highsequency boosting (512 by 512 pixels).

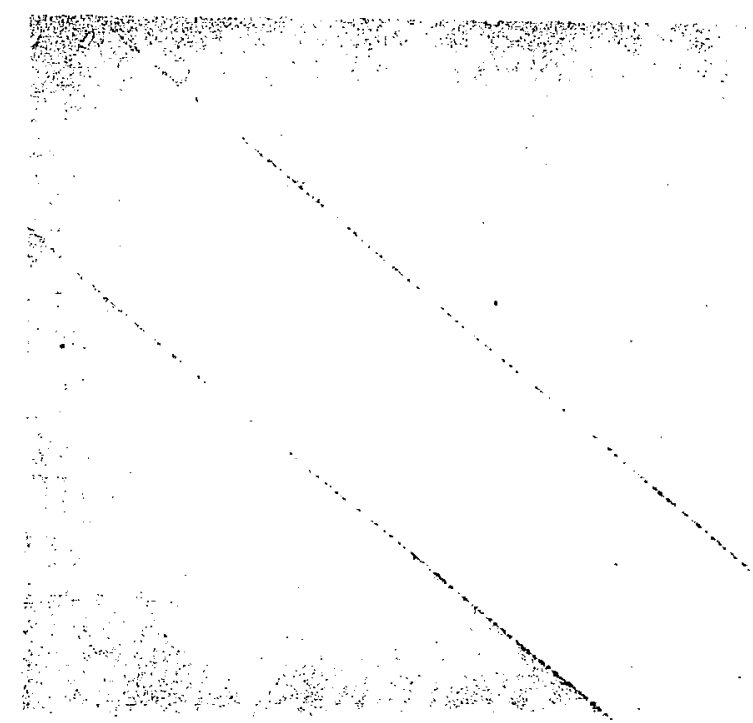

Fig. 6. Step tablet image after highsequency bcosting (512 by 512 pixels). 


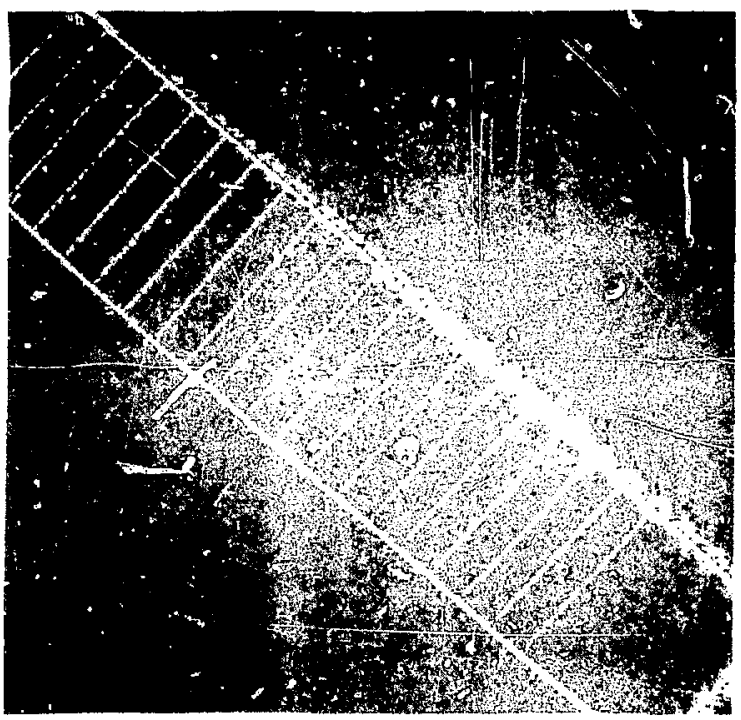

Fig. 7. Step tablet image after high-sequency boosting and adaptive filtering (512 by 512 pixe1s).

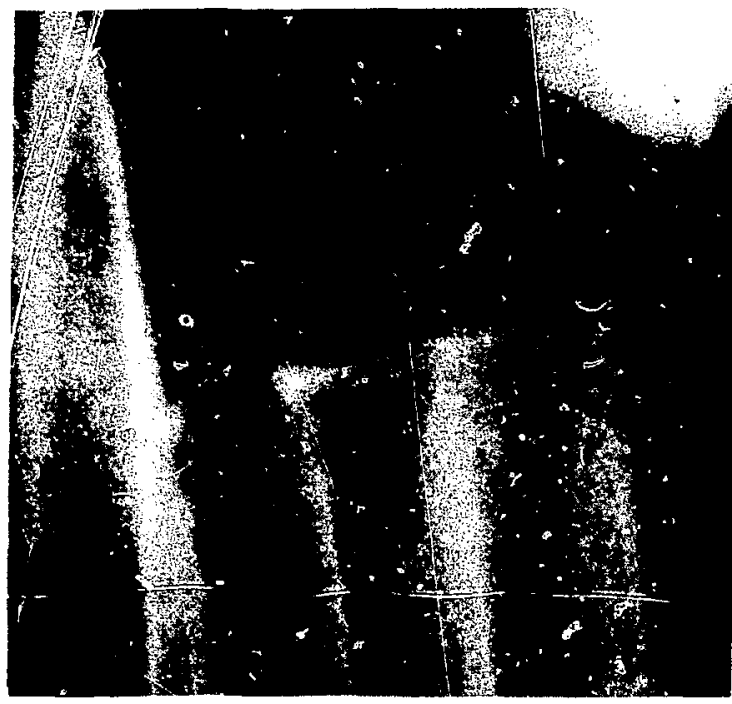

Fig. 8. PHERMEX flash radiographic image (512 by 512 pixe]s).

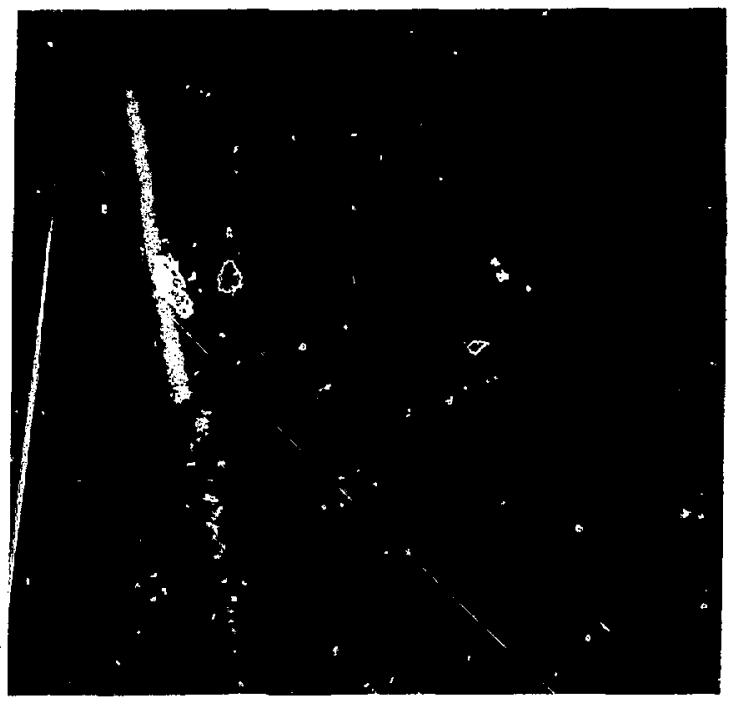

Fig. 9. PHERMEX image after histogram equalization, Haar highsequency boosting, and adaptive filtering. 